\title{
Computational prediction of miRNA binding sites in mRNA of colorectal cancer candidate genes
}

\author{
Aigul Akimniyazova \\ SRI of Biology and Biotechnology problems \\ al-Farabi Kazakh National University \\ Almaty, Kazakhstan \\ email: akimniyazova@gmail.com
}

\begin{abstract}
The identification of causative miRNAs in colorectal cancer is unclear due to a lack of understanding of how specific miRNAs affect biological pathways and consequences of disease development. The miRNA is a class of nano-sized non-coding RNAs that regulate many mRNAs, and mRNA can be related to many miRNAs, which make them suitable for diagnosis purposes. Therefore it is required to identify candidate genes of colorectal cancer and to what extent they can interact with miRNA. To determine the important miRNAs biding sites in genes, involved in the development of colorectal cancer, there were used the MirTarget program. The paper presents the results of studying the characteristics of the interaction of miRNAs with mRNAs of 135 candidate genes involved in the development of colorectal cancer. The binding sites of 446 miRNAs have in 113 mRNA of genes at 5'UTR, CDS, and 3'UTR. Found miRNA binding sites with overlapped nucleotide sequences (clusters). The research results are useful for the development of methods for early diagnosis of this disease.
\end{abstract}

Keywords - miRNA, mRNA, genes, colorectal cancer

\section{Introduction}

Colorectal cancer is one of the three most common oncological diseases worldwide and has a high mortality rate [1-2]. The complexity of early diagnosis of the disease lies in its polygenic nature. Colorectal cancer is accompanied by a change in the concentration of miRNAs, which can alter the expression of candidate genes related with the disease. The effect of miRNA on cancerogenesis is described, however it has not been established which target genes these miRNAs can directly affect [3-4]. The purpose of this study was to identify the interactions between human miRNAs and 135 colorectal cancer candidate gene mRNAs.

\section{Materials and Methods}

The nucleotide (nt) sequences of 135 colorectal cancer candidate genes were downloaded from GenBank (http://www.ncbi.nlm.nih.gov). The nucleotide sequences of 2,565 miRNAs were taken from miRBase, and 3,707 miRNAs from a previous study [5]. The quantitative characteristics of these interactions were determined using the MirTarget program created in our laboratory [6-7]. This program defines the start of miRNA binding sites in mRNA; localization of binding sites in 5'-untranslated region (5'UTR), protein coding region (CDS), and 3'-untranslated region (3'UTR); free energy of interaction $(\Delta \mathrm{G}, \mathrm{kJ} / \mathrm{mole})$ and scheme of miRNAmRNA nucleotides (nt) interaction. The $\Delta \mathrm{G} / \Delta \mathrm{Gm}(\%)$ ratio was calculated for each binding site, where $\Delta \mathrm{Gm}$ is equal to the free energy of miRNA interaction with fully complementary nucleotide sequence.

\section{Results}

The binding sites of 446 miRNAs in 113 candidate gene mRNAs were determined considering their expression. Significant differences were found in the characteristics of the
miRNA interactions in the 5'UTR, CDS and 3'UTR of mRNA candidate genes. The features of the miRNA binding sites have been established depending on their location in the 5'UTR, CDS and 3'UTR. The miRNA binding sites with overlapping nucleotide sequences that form clusters were identified. This organization of binding sites leads to compaction and competition between miRNAs for binding in the cluster. The most effective associations between miRNAs and candidate target genes, which are proposed as markers for the development of methods for the early diagnosis of colorectal cancer, are determined. Studying the interactions between miRNAs and mRNAs was carried out with colorectal cancer candidate genes with RPKM (Reads per kilo base per million mapped reads) expression values up to ten and greater than ten, considering the localization of miRNA binding sites in the 5 'UTR, CDS or 3'UTR. The adequate prediction of miRNA binding sites in target gene mRNAs is a key problem for studying the role of miRNAs in the regulation of gene expression.

Characteristics of miRNA interactions with $5^{\prime} U T R$ $m R N A s$ of candidate colorectal cancer genes.

Five binding sites in 5'UTR in mRNA of DCC gene was revealed. The mRNA of FMNL3 gene was target of four miRNAs, three of them forms the cluster of binding sites from $51 \mathrm{nt}$ to $77 \mathrm{nt}$ with an average $\Delta \mathrm{G}$ value equal to $-124 \mathrm{~kJ} / \mathrm{mole}$. ID01310.3p-miR and ID03332.3p-miR forms a cluster of binding sites in mRNA of KRAS gene in position from $17 \mathrm{nt}$ to 60 nt. SMAD2, SMAD4 and SOCS6 genes had binding sites for miRNAs were located along the entire length of the 5'UTR mRNA of genes without overlapping nucleotide sequences. The miR-6786-5p and ID03064.3p-miR binding sites in the mRNA of the SMAD7 gene overlap on seven nucleotides, which makes simultaneous binding of these miRNAs impossible. ID03064.3p-miR has the advantage of binding to mRNA, since it has a large free interaction energy compared to miR-6786-5p. mRNA of CD44 and CTNNB1 were a targets of two miRNAs for each, that contain independent from each other binding sites that not overlap their nucleotide sequence. ID00894.5p-miR and ID02781.3p-miR forms a cluster of binding sites in mRNA of $C D H 1$ gene from $57 \mathrm{nt}$ to $79 \mathrm{nt}$. The mRNA of FN1 gene had miRNA binding sites that were located in 5'UTR and CDS. These binding sites did not overlap, and each miRNA could independently bind to the mRNA of the FN1 gene. mRNA of GSTP1 gene was target only of two miRNAs (ID00267.3p-miR and ID03331.3p$\mathrm{miR}$ ), that form a cluster from $77 \mathrm{nt}$ to $113 \mathrm{nt}$ with an average free energy of interaction equal to $-122 \mathrm{~kJ} / \mathrm{mole}$. miR-6789$5 \mathrm{p}$, ID01675.5p-miR and ID02822.5p-miR forms cluster of binding sites in mRNA of HIF $1 A$ gene from $54 \mathrm{nt}$ to $89 \mathrm{nt}$ with an average $\Delta \mathrm{G}$ value equal to $-127 \mathrm{~kJ} /$ mole. miR-6789-5p in this cluster have a prevalent chance to take this binding site because of higher free energy of interaction equal to -132 $\mathrm{kJ} /$ mole and $\Delta \mathrm{G} / \Delta \mathrm{Gm}$ value equal to $90 \%$. The mRNAs of SFRP2, STAT3 and TGFBR1 genes had clusters of miRNA 
binding sites. ID01777.3p-miR, ID00849.3p-miR and ID01545.3p-miR forms cluster of binding sites in mRNA of $Z E B 1$ gene from $1 \mathrm{nt}$ to $28 \mathrm{nt}$ with an average $\Delta \mathrm{G}$ equal to $116 \mathrm{~kJ} /$ mole.

Characteristics of miRNA interactions with CDS $m R N A s$ of candidate colorectal cancer genes. mRNAS of ADAMTS4, BCL2, BIRC5, CYP1B1, DNMT1, ESR1, KSR1, MSH6, PIK3CG, SMAD7 and TNFRSF4 genes had binding sites only for two miRNAs in CDS. 21 miRNAs form a cluster of binding sites $\mathrm{n}$ mRNA of $G S K 3 B$ gene. The $G S K 3 B$ gene mRNA included a cluster of binding sites ranging from $3 \mathrm{nt}$ to $38 \mathrm{nt}$, with an entire length of $36 \mathrm{nt}$ and an average $\Delta \mathrm{G}$ of $-126 \mathrm{~kJ} / \mathrm{mole}$. These 22 binding sites have a total length equal to $750 \mathrm{nt}$, which is 20 times larger than the cluster length. The formation of these clusters of binding sites in the $G S K 3 B$ gene 5'UTR indicates the greater ability of this gene for compaction, which causes competition between the given miRNAs for the binding site. The mRNA of ONECUT2, PIK3CA and TCF4 also found big cluster of binding sites. These binding sites did not overlap and each miRNA could independently bind to mRNA of given genes. ID01242.3p-miR, ID00306.5p-miR and miR$511-5 \mathrm{p}$ had binding sites in mRNA of ADAMTS5 gene. mRNA of $O G G 1$ gene was target of three miRNAs, two of which form the cluster of binding sites from $443 \mathrm{nt}$ to $462 \mathrm{nt}$. CDS mRNA of POLD1 gene contain binding sites for five miRNAs, among them, ID03114.3p-miR and miR-629-3p forms a cluster of binding sites from 1069 nt to $1094 \mathrm{nt}$ with an average $\Delta \mathrm{G}=-112 \mathrm{~kJ} / \mathrm{mole}$. Three miRNAs had binding sites in CDS mRNA of POLE gene. mRNA of $A L D H 2$ gene contains four miRNAs binding sites, three of them form a cluster in position from $153 \mathrm{nt}$ to $191 \mathrm{nt}$ with an average $\Delta \mathrm{G}$ value equal to $-115 \mathrm{~kJ} / \mathrm{mole}$. Four miRNAs had binding sites in mRNA of CDH1 gene. miR-6798-5p, miR-3909 and ID02852.5p-miR had binding sites in mRNA of COL3A1 gene. mRNA of ERBB2 gene have a cluster of binding sites from $3815 \mathrm{nt}$ to $3838 \mathrm{nt}$ with an average $\Delta \mathrm{G}=-116 \mathrm{~kJ} / \mathrm{mole}$. mRNA of $H M G B 1$ gene was target of three miRNAs, two of which forms a cluster from $746 \mathrm{nt}$ to $770 \mathrm{nt}$. ID01524.3p-miR and ID03238.3p-miR form a cluster of binding sites in mRNA of MTA1 gene from $406 \mathrm{nt}$ to $444 \mathrm{nt}$ with an average $\Delta \mathrm{G}=$ $115 \mathrm{~kJ} /$ mole and also have binding sites for ID01593.5p-miR and ID01702.3p-miR in CDS. ID00645.5p-miR and miR$6752-5 p$ form a cluster of binding sites in mRNA of $M U C 1$ gene from $507 \mathrm{nt}$ to $531 \mathrm{nt}$ with an average $\Delta \mathrm{G}=-114$ $\mathrm{kJ} /$ mole. mRNA of SEPT9 gene was target of three miRNAs, two of which form a cluster of binding sites from $1350 \mathrm{nt}$ to 1381 nt. SFRP2, THBS2 and VEGFA had binding sites for two miRNAs.

Characteristics of miRNA interactions with 3'UTR $m R N A$ s of candidate colorectal cancer genes

The miR-1273g-3p and miR-1273d had binding sites in mRNA of $A L O X 15$ gene in 3'UTR. These binding sites did not overlap, and each miRNA could independently bind to this mRNA. The mRNA of FMNL3 gene was target of four miRNAs. The mRNA of KRAS gene was a target of four miRNAs, two of which form a cluster of binding sites from
$3163 \mathrm{nt}$ to $3176 \mathrm{nt}$ with an average $\Delta \mathrm{G}$ value equal to -115 $\mathrm{kJ} /$ mole. Five miRNAs had binding sites in mRNA of MTAP gene, and among them ID01836.5p-miR and miR-1285-5p form a cluster of binding sites in position from $2530 \mathrm{nt}$ to 2566 nt with an average $\Delta \mathrm{G}=-112 \mathrm{~kJ} /$ mole. mRNA of BCL2L1 gene was a target of two miRNAs in 3'UTR. miR-1273c and miR-1273g-3p form a cluster of binding sites in position from $3251 \mathrm{nt}$ to $3291 \mathrm{nt}$. miR-619-5p has a binding sites in mRNA of $E R B B 3$ gene. Previously we investigated the variation of nucleotide sequences of these two miR-619-5p binding sites in the 3'UTR of mRNAs of ERBB3 gene in orthologs [8]. It is found that the binding site with $100 \%$ score contains highly conserved nucleotide sequence GGCTCATGCCTGTAATCCCAGC of miR-619-5p binding sites. The mRNA of FASN and GREMI contains by two binding sites for miRNAs in 3'UTR. ID00791.5p-miR and miR-6829-5p forms a cluster of binding sites in MTA1 gene from $2360 \mathrm{nt}$ to $2392 \mathrm{nt}$ with an average $\Delta \mathrm{G}=-116 \mathrm{~kJ} / \mathrm{mole}$. Four miRNAs had binding sites in mRNA of PIK3CG gene. The miR-1277-5p had three binding sites that does not overlap in mRNA of $V E G F A$ gene.

\section{Conclusion}

It was found that miRNA binding sites in mRNA of target genes were located in all mRNA regions. Clusters of miRNA binding sites have been identified in some genes. The obtained results show that the interaction of the miRNAs with mRNAs can serve as the basis for the selection of associations for the diagnosis of colorectal cancer.

\section{ACKNOWLEDGMENT}

The work was performed within the framework of the grant project AP05132460 financed by the Ministry of Education and Science of the Republic of Kazakhstan

\section{REFERENCES}

[1] F. Bray, J. Ferlay, I. Soerjomataram, RL. Siegel, LA. Torre, and A. Jemal, "Global cancer statistics 2018: GLOBOCAN estimates of incidence and mortality worldwide for 36 cancers in 185 countries," CA Cancer J Clin., vol. 68(6), pp. 394-424, November 2018.

[2] M. Arnold, MS. Sierra, M. Laversanne, I. Soerjomataram, A. Jemal, F. Bray, "Global patterns and trends in colorectal cancer incidence and mortality," Gut, vol. 66, pp. 683-91, 2017.

[3] B. Chen, Z. Xia, YN.Deng, et al, "Emerging microRNA biomarkers for colorectal cancer diagnosis and prognosis," Open Biol., vol. 9(1), 2019.

[4] A.W. Tong and J. Nemunaitis, "Modulation of miRNA activity in human cancer: a new paradigm for cancer gene therapy?" Cancer Gene Therapy, vol. 15, pp. 341-355, 2008

[5] E. Londin, P. Loher, A.G. Telonis, K. Quann, P. Clark, Y. Jing et al, "Analysis of 13 cell types reveals evidence for the expression of numerous novel primate- and tissue-specific microRNAs," PNAS USA, vol. 112, pp. 1106-1115.

[6] A. Ivashchenko, O. Berillo, A. Pyrkova, R. Niyazova, Sh. Atambayeva, "The Properties of Binding Sites of miR-619-5p, miR-5095, miR-5096, and miR-5585-3p in the mRNAs of Human Genes," BioMed research international, 2014

[7] A. Ivashchenko, O. Berillo, A. Pyrkova, R. Niyazova, "Binding Sites of miR-1273 Family on the mRNA of Target Genes," BioMed research international, 2014.

[8] S. Atambayeva, R. Niyazova, A. Ivashchenko, A. Pyrkova, I. Pinsky, A. Akimniyazova, S. Labeit, "The Binding Sites of miR-619-5p in the mRNAs of Human and Orthologous Genes," BMC Genomics, vol. 18, pp. 428. 